\title{
An existence result for evolution equations in non-cylindrical domains
}

\author{
Fabio Paronetto
}

\begin{abstract}
We give an existence and uniqueness result for an evolution equation $u_{t}+\mathcal{A} u=f$, with suitable boundary data and where $\mathcal{A}$ is a strictly monotone operator, in a non-cylindrical domain.
\end{abstract}

Mathematics Subject Classification. 35.01, 35K22, 35K55, $47 \mathrm{H} 05$.

Keywords. Parabolic equations, Non-cylindrical domain, Monotone operators.

\section{Introduction}

In this note we want to consider evolution problems in a non-cylindrical domain, as for instance

$$
\begin{cases}\frac{\partial u}{\partial t}-\Delta_{x} u=f & \text { in } Q \\ u(x, t)=0 & \text { in } \Gamma \\ u(x, 0)=u_{o}(x) & \text { in } \Omega(0),\end{cases}
$$

where $\Delta_{x}:=\sum_{i=1}^{n} \frac{\partial}{\partial x_{i}}, Q$ is an open subset of $A \times[0, T]$ where $A$ is a subset of $\mathbf{R}^{n}\left(Q\right.$ is $\cup_{t \in(0, T)} \Omega(t)$ for some open and non-empty $\left.\Omega(t) \subset A\right)$, the variable in $Q$ is denoted by $(x, t)$ meaning in fact $\left(x_{1}, \ldots x_{n}, t\right)$, and $\Gamma$ is $\cup_{t \in(0, T)} \partial \Omega(t)$ and $u_{o}$ and $f$ are suitable data.

This problem has been considered by many authors and can be attacked in different ways. We confine to quote a few papers trying to draw the attention to the different techniques. A first paper we quote is [4], where the authors suppose the existence of a $C^{2}$-diffeomorphism between the non-cylindrical set and a proper cylinder in $\mathbf{R}^{n+1}$.

As far as we know other techniques are the following: the problem can be studied via semigroup theory, in which one can see the equation as $u^{\prime}(t)+$ $A(t) u(t)=f(t)$ for $t>0$ where $A(t)$ is an operator whose domain $D_{A(t)}$ depends on $t$ and may change with $t$ (see, for instance [2] and the references therein); can be solved taking the perturbed equation $u_{t}-\Delta_{x} u-\varepsilon u_{t t}=f$ and then study the limit behaviour of a suitable problem for $\varepsilon \rightarrow 0^{+}$(see, for 
instance $[5,9])$; another way of seeing this problem is via minimizing movements (see, for instance [7]).

Again, as far as we know, about the variation with respect to $t$ of the open sets $\Omega(t)$ 's it is required the already mentioned regularity in [4], a Lipschitz or Hölder continuity (as required in [5]) an increasing monotonicity, i.e. $\Omega(s) \subseteq \Omega(t)$ for $s<t$ (as required in [7]).

Another paper we mention is [1], where the porous medium equation is considered in a non-cylindrical domain.

In this paper our purpose is to consider the problem in a different way, a technique by which we weaken the requirements, at least those mentioned above. We do not look at the equation as an abstract evolution equation in the variable $t$, but simply as an equation in a domain of $\mathbf{R}^{n+1}$. As regards the assumptions, we do not require monotonicity on $\Omega(t)$ 's and about regularity we assume the variation of $\Omega(t)$ to be an absolute continuous function in time and not to be Lipschitz or Hölder continuous (see (H.2) below for more details).

Finally we do not confine to consider linear operators, but we consider

$$
\begin{cases}\frac{\partial u}{\partial t}+\mathcal{A} u=f & \text { in } Q \\ u(x, t)=0 & \text { in } \Gamma \\ u(x, 0)=u_{o}(x) & \text { in } \Omega(0)\end{cases}
$$

with $\mathcal{A}$ monotone operator; for example $\mathcal{A} u=-\operatorname{div}\left(|D u|^{p-2} D u\right), p \geq 2$ is admitted.

\section{Assumptions and preliminary results}

Consider $p \geq 2, T>0$ and $A$ an open and bounded subset of $\mathbf{R}^{n}$, for simplicity suppose moreover $A$ connected. Consider $\hat{Q} \subset A \times[0, T]$ a measurable subset of $\mathbf{R}^{n+1}$ in such a way that

$\Omega(t):=\hat{Q} \cap(A \times\{t\})$ is an open, connected subset of $\mathbf{R}^{n}$ for every $t \in(0, T)$.

In this way we have

$$
\hat{Q}=\cup_{t \in[0, T]} \Omega(t)
$$

moreover we define

$$
Q=\cup_{t \in(0, T)} \Omega(t), \quad \Gamma:=\cup_{t \in(0, T)} \partial \Omega(t) .
$$

Notice that the set $\Omega(t)$ is measurable, at least for almost every $t \in[0, T]$.

Remark 2.1. Notice that we are not requiring $\Omega(0) \neq \emptyset$ or $\Omega(0)$ connected. This means that the sets $\Omega(0)$ and $\Omega(T)$ could be in principle not connected and also empty: for example $\bar{Q} \cap(\bar{A} \times\{0\})$ could be a closed set whose inner part is empty. Moreover $\Omega(t)$ in fact can be also disconnected, but we confine to consider $\Omega(t)$ connected and will show in the last section with some examples how $\Omega(t)$ can be disconnected. 
In fact the set $\Omega(t)$ will turn out to be measurable for every $t \in[0, T]$. Indeed about the family of open sets $\Omega(t)$ we will make the following assumptions:

(H.1) $\Omega(t)$ is a subset of $A$ for every $t \in[0, T], A$ being a bounded open subset of $\mathbf{R}^{n}$, and $\Omega(t)$ is an open, connected and not empty set with Lipschitz boundary for almost every $t \in[0, T]$;

(H.2) we suppose that for every $t_{o} \in[0, T]$ there exists a family of maps depending on the parameter $t \in I_{t_{o}} \cap[0, T]$ where $I_{t_{o}}$ is an open neighbourhood of $t_{o}$

$$
G_{t}^{t_{o}}=G^{t_{o}}(\cdot, t): \Omega\left(t_{o}\right) \rightarrow \Omega(t)
$$

such that the map $G$ satisfies

- $G^{t_{o}}(\cdot, t)$ is a bijection for almost every $t \in I_{t_{o}}$,

- $G^{t_{o}}(\cdot, t)$ is Lipschitz continuous with its inverse for every $t \in I_{t_{o}}$

- $G^{t_{o}}(x, \cdot)$ and $\left|D G^{t_{o}}(x, \cdot)\right|$ are absolutely continuous for almost every $x \in \Omega\left(t_{o}\right)$,

- $\left|D G^{t_{o}}(\cdot, t)\right| \in L^{1}\left(\Omega\left(t_{o}\right)\right)$ for every $t$ and $\frac{\partial}{\partial t}\left|D G^{t_{o}}\right| \in L^{1}\left(\Omega\left(t_{o}\right) \times\right.$ $(0, T))$,

where $\left|D G^{t_{o}}(\cdot, t)\right|=\left|D G_{t}^{t_{o}}\right|$ denotes the absolute value of determinant of the Jacobian matrix of $G_{t}^{t_{o}}$.

Comments about assumptions Assumption (H.1) is simply the request that $\Omega(t)$ is a an open set with Lipschitz boundary. One could probably weaken the assumption about the regularity of $\partial \Omega(t)$, but our main goal is not this. We stress that (H.1) may hold for almost every $t$, i.e. it could fail for some $t$ 's. We have in mind some particular cases which will be explained in the last section. The meaning of (H.2) is essentially the following: we want that $\Omega(t)$ is a regular deformation of its neighbours, at least for almost every $t$. By regular, and this is the important thing, we mean that $G^{t_{o}}(x, \cdot)$ and $\left|D G^{t_{o}}(x, \cdot)\right|$ are absolutely continuous with respect to $t$ (see the first example in the last section). These requirements about these two functions ensure the absolute continuity, and then the differentiability, of the two functions

$$
t \mapsto \int_{\Omega(t)} d x, \quad t \mapsto \int_{\Omega(t)} w(x, t) d x \quad \text { with } w \in C^{1}(\bar{Q})
$$

as shown below to obtain formula (4). Thanks to density of regular functions in the space $\mathcal{W}$ defined below, in which the solution of problem (1) lives, and thanks to the boundary conditions $(u=0$ in $\Gamma)$ we obtain the differentiability of the function $t \mapsto \int_{\Omega(t)} u(x, t) v(x, t) d x$ for $u, v \in \mathcal{W}$.

We continue now giving some definitions and preliminary results. We consider $C_{c}^{1}(Q)$, the set of $C^{1}$ functions defined in $Q$ and whose support is contained in $Q$. For a function $v$ belonging to $C_{c}^{1}(Q)$ we denote the gradient of $v$ as follows

$$
\operatorname{grad} v=\left(D_{1} v, \ldots, D_{n} v, v_{t}\right):=\left(D v, v_{t}\right)
$$


where $D v$ is the vector in $\mathbf{R}^{n}$ of the first $n$ derivatives. For $v \in C_{c}^{1}(Q)$ consider the quantity

$$
N(v):=\left(\iint_{Q}|D v|^{p} d x d t\right)^{1 / p} .
$$

It is immediate to verify that $N$ is a norm: indeed if $v \in C_{c}^{1}(Q)$ and $N(v)=0$ we have that $v$ is a function depending only on $t$. We want to show that $v \equiv 0$ in $I$, where $I$ is the projection in $[0, T]$ of $Q$. Notice that $I$ is also the projection of $\partial Q$ and since $v=0$ in $\partial Q$ we get that $v(t)=0$ for every $t \in I$, i.e. $v \equiv 0$. For $v \in C_{c}^{1}(Q)$ define the two norms

$$
\|v\|_{1}:=\left(\iint_{Q}\left[|v|^{p}+|D v|^{p}\right] d x d t\right)^{1 / p}, \quad\|v\|_{2}:=\left(\iint_{Q}|D v|^{p} d x d t\right)^{1 / p} .
$$

Lemma 2.2. The two norms $\|\cdot\|_{1}$ and $\|\cdot\|_{2}$ are equivalent in $C_{c}^{1}(Q)$.

Proof. The only thing to verify is that there is a positive constant $c$ such that

$$
\|v\|_{1} \leq c\|v\|_{2} \text { for every } v \in C_{c}^{1}(Q) .
$$

Since $v(\cdot, t) \in C_{c}^{1}(\Omega(t))$ we have (see, e.g. [8, section 7.8])

$$
\int_{\Omega(t)}|v(x, t)|^{p} d x d t \leq\left(\frac{|\Omega(t)|}{\omega_{n}}\right)^{p / n} \int_{\Omega(t)}|D v(x, t)|^{p} d x d t
$$

where $\omega_{n}$ denotes the measure in $\mathbf{R}^{n}$ of the ball of radius 1 and since

$$
\iint_{Q}|v(x, t)|^{p} d x d t=\int_{0}^{T} \int_{\Omega(t)}|v(x, t)|^{p} d x d t
$$

we conclude

$$
\iint_{Q}|v|^{p} d x d t \leq\left(\frac{1}{\omega_{n}}\right)^{p / n}\left(\sup _{t \in(0, T)}|\Omega(t)|\right)^{p / n} \iint_{Q}|D v|^{p} d x d t .
$$

Now we define the spaces

$$
\mathcal{H} \text { and } \mathcal{V}
$$

respectively as the completion of $C_{c}^{1}(Q)$ or alternatively of the space $\mathcal{U}$ defined by

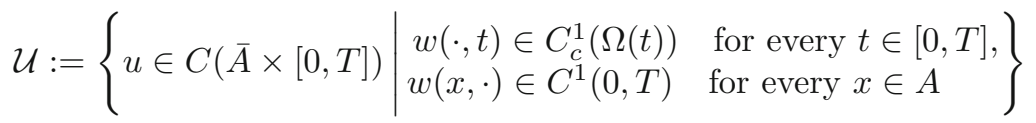

with respect to the following norms

$$
\|u\|_{\mathcal{H}}:=\left(\iint_{Q} u^{2}(x, t) d x d t\right)^{1 / 2}, \quad\|u\|_{\mathcal{V}}:=\left(\iint_{Q}|D u|^{p}(x, t) d x d t\right)^{1 / p}
$$


and $\mathcal{V}^{\prime}$ as the dual space of $\mathcal{V}$. We will consider $p \geq 2$ in such a way that $\mathcal{V} \subset \mathcal{H}$ and since $\Omega(t) \subset A$ for every $t \in(0, T)$ by the previous lemma and Hölder's inequality we get

$$
\|u\|_{\mathcal{H}} \leq(|A| T)^{\frac{p-2}{2 p}}\left(\frac{|A|}{\omega_{n}}\right)^{1 / n}\|u\|_{\mathcal{V}} .
$$

We also define the Banach space

$$
\mathcal{W}:=\left\{u \in \mathcal{V} \mid u_{t} \in \mathcal{V}^{\prime}\right\}, \quad\|u\|_{\mathcal{W}}:=\|u\|_{\mathcal{V}}+\left\|u_{t}\right\|_{\mathcal{V}^{\prime}}
$$

where by $u_{t}$ we denote the distributional derivative with respect to time $D_{t} u$ defined by

$$
\iint_{Q} u \frac{\partial \phi}{\partial t} d x d t=-\left\langle D_{t} u, \phi\right\rangle=:-\left\langle u_{t}, \phi\right\rangle \quad \text { for every } \phi \in C_{c}^{\infty}(Q) .
$$

Since the embedding $\mathcal{V} \subset \mathcal{H}$ is continuous and dense and identifying $\mathcal{H}^{\prime}$ with $\mathcal{H}$ we have

$$
\mathcal{V} \subset \mathcal{H} \subset \mathcal{V}^{\prime}
$$

with continuous embeddings.

Notice that if we consider the following family of evolution triplets

$$
V(t) \subset H(t) \subset V(t)^{\prime} \quad t \in[0, T]
$$

where

$$
V(t)=W_{0}^{1, p}(\Omega(t)), \quad H(t)=L^{2}(\Omega(t)), \quad V^{\prime}(t)=W^{-1, p^{\prime}}(\Omega(t))
$$

for every $t \in(0, T)$, then the spaces $\mathcal{V}$ and $\mathcal{H}$ defined above can be also seen as the closure of $\mathcal{U}$ with respect to the following norms

$$
\|v\|_{\mathcal{V}}^{p}:=\int_{0}^{T}\|v(t)\|_{V(t)}^{p} d t \quad \text { and }\|v\|_{\mathcal{H}}^{2}:=\int_{0}^{T}\|v(t)\|_{H(t)}^{2} d t
$$

since the functions $t \mapsto\|v(t)\|_{V(t)}^{p}$ and $t \mapsto\|v(t)\|_{H(t)}^{2}$ turn out to be measurable and where by $v(t)$ we denote the function $v(\cdot, t)$. Because of that we will sometimes write

$$
v(t) \text { to denote the function } v(\cdot, t) \text {. }
$$

Lemma 2.3. The space $\mathcal{U}$ is dense in the space $\mathcal{W}$.

Proof. Consider $\partial Q=\Gamma \cup \partial \Omega(0) \cup \Omega(0) \cup \partial \Omega(T) \cup \Omega(T)$. Any function $u \in \mathcal{U}$ vanishes in $\hat{\Gamma}:=\Gamma \cup \partial \Omega(0) \cup \partial \Omega(T)$ and so the only part of the boundary in which may be not zero is $\Omega(0) \cup \Omega(T)$. This part of the boundary clearly satisfies the segment property (see, e.g., Theorem 3.18 in [3]) and then the space $\mathcal{U}$ is dense in

$$
W_{\hat{\Gamma}}^{1, p}(Q)=\left\{u \in L^{p}(Q)\left|D_{j} u \in L^{p}(Q), j=1, \ldots n, D_{t} u \in L^{p}(Q), u\right|_{\hat{\Gamma}}=0\right\}
$$

and, by the density of $L^{p}(Q)$ in $\mathcal{V}^{\prime}$, this is dense in

$$
\left\{u \in L^{p}(Q)\left|D_{j} u \in L^{p}(Q), j=1, \ldots n, D_{t} u \in \mathcal{V}^{\prime}, u\right|_{\hat{\Gamma}}=0\right\}=\mathcal{W} .
$$


Before going on we recall a couple of results (formula (3) and the following lemma).

Lemma 2.4. Consider $E$ a measurable subset of $\mathbf{R}^{n}$, a measurable function $f: E \times[0, T] \rightarrow \mathbf{R}$ such that $t \mapsto f(x, t)$ is absolutely continuous for almost every $x \in E, f(\cdot, t) \in L^{1}(E)$ for every $t \in[0, T]$ and $\frac{\partial f}{\partial t} \in L^{1}(E \times(0, T))$. Then we have that the function $t \mapsto \int_{E} f(x, t) d x$ is absolutely continuous in $[0, T]$ and

$$
\left.\frac{d}{d t}\right|_{t=s} \int_{E} f(x, t) d x=\int_{E} \frac{\partial f}{\partial t}(x, s) d x
$$

for almost every $s \in(0, T)$.

Proof. Once called $F$ the function $F(t)=\int_{E} f(x, t) d x$ one has, by the absolute continuity of $f$ with respect to time,

$$
\begin{aligned}
\lim _{h \rightarrow 0} \frac{F(s+h)-F(s)}{h} & =\lim _{h \rightarrow 0} \frac{1}{h} \int_{E}[f(x, s+h)-f(x, s)] d x \\
& =\lim _{h \rightarrow 0} \frac{1}{h} \int_{E}\left[\int_{s}^{s+h} \frac{\partial f}{\partial t}(x, t) d t\right] d x \\
& =\lim _{h \rightarrow 0} \frac{1}{h} \int_{s}^{s+h}\left[\int_{E} \frac{\partial f}{\partial t}(x, t) d x\right] d t=\int_{E} \frac{\partial f}{\partial t}(x, s) d x
\end{aligned}
$$

provided that $s \in(0, T)$ is a Lebesgue's point for the function $t \mapsto$ $\int_{E} \frac{\partial f}{\partial t}(x, t) d x$ and $t \mapsto f(x, t)$ is differentiable in $s$.

With assumptions (H.1) and (H.2) we get that the function $t \mapsto \int_{\Omega(t)} d x$ is absolutely continuous and then differentiable and using Lemma 2.4 the following hold

$$
\begin{aligned}
\left.\frac{d}{d t}\right|_{t=t_{o}} & \int_{\Omega(t)} d x=\left.\frac{d}{d t}\right|_{t=t_{o}} \int_{\Omega\left(t_{o}\right)}|D G(x, t)| d x=\int_{\Omega\left(t_{o}\right)} \frac{\partial|D G|}{\partial t}\left(x, t_{o}\right) d x, \\
& \int_{\Omega\left(t_{2}\right)} d x-\int_{\Omega\left(t_{1}\right)} d x=\int_{t_{1}}^{t_{2}} \int_{\Omega(s)} \frac{\partial|D G|}{\partial t}(x, s) d x d s .
\end{aligned}
$$

To compute the quantity in the right hand side of this last equality one can use the formula

$$
\frac{d}{d t}(\operatorname{det} A(t))=\operatorname{det} A(t) \operatorname{tr}\left(A^{-1}(t) \frac{d}{d t} A(t)\right),
$$

where $A(t)$ is a matrix whose coefficients depend on the parameter $t$. More in general, given $w \in C^{1}(\bar{Q})$, using the change of variable $G_{t}^{t_{o}}$ and formula (3) by a direct computation one gets (see also [6]) 


$$
\begin{aligned}
\left.\frac{d}{d t}\right|_{t=t_{o}} \int_{\Omega(t)} w(y, t) d y=\left.\frac{d}{d t}\right|_{t=t_{o}} \int_{\Omega\left(t_{o}\right)} w(G(x, t), t)|D G(x, t)| d x \\
=\int_{\Omega\left(t_{o}\right)} \sum_{i=1}^{n} \frac{\partial w}{\partial y_{i}}\left(G\left(x, t_{o}\right), t_{o}\right) \frac{\partial G_{i}}{\partial t}\left(x, t_{o}\right)\left|D G\left(x, t_{o}\right)\right| d x \\
\quad+\int_{\Omega\left(t_{o}\right)} \frac{\partial w}{\partial t}\left(G\left(x, t_{o}\right), t_{o}\right)\left|D G\left(x, t_{o}\right)\right| d x \\
\quad+\left.\int_{\Omega\left(t_{o}\right)} w(G(x, t), t) \frac{\partial}{\partial t}\right|_{t=t_{o}}|D G(x, t)| d x \\
=\int_{\Omega\left(t_{o}\right)} \frac{\partial w}{\partial t}\left(y, t_{o}\right) d y+\int_{\Omega\left(t_{o}\right)} \operatorname{div}\left(w\left(y, t_{o}\right) V\left(y, t_{o}\right)\right) d y \\
=\int_{\Omega\left(t_{o}\right)} \frac{\partial w}{\partial t}\left(y, t_{o}\right) d y+\int_{\partial \Omega\left(t_{o}\right)} w\left(y, t_{o}\right) V\left(y, t_{o}\right) \cdot \nu\left(y, t_{o}\right) d \mathscr{H}^{n-1}(y)
\end{aligned}
$$

where $\nu\left(\cdot, t_{o}\right)$ is the external normal (where it exists) to $\Omega\left(t_{o}\right), d \mathscr{H}^{n-1}$ denotes the Hausdorff $(n-1)$-dimensional measure and $V$ is a vector field whose components are given by $V_{i}(y, t)=\frac{\partial G_{i}}{\partial t}\left(G_{t}^{-1}(y), t\right)$. In particular taking $w \in \mathcal{U}$ we get

$$
\left.\frac{d}{d t}\right|_{t=t_{o}} \int_{\Omega(t)} w(x, t) d x=\int_{\Omega\left(t_{o}\right)} \frac{\partial w}{\partial t}\left(x, t_{o}\right) d x .
$$

Using this formula with $w=u v$ with $u, v \in \mathcal{U}$ we get

$$
\int_{t_{1}}^{t_{2}} \int_{\Omega(t)} \frac{\partial}{\partial t}(u v) d x d t=\int_{\Omega\left(t_{2}\right)} u\left(x, t_{2}\right) v\left(x, t_{2}\right) d x-\int_{\Omega\left(t_{1}\right)} u\left(x, t_{1}\right) v\left(x, t_{1}\right) d x .
$$

Now notice that in fact for $u, v \in \mathcal{U}$ we have

$$
(u(t), v(t))_{H(t)}=(u(t), v(t))_{L^{2}(\Omega(t))}=(u(t), v(t))_{L^{2}(A)} .
$$

Proposition 2.5. For every $u, v \in \mathcal{W}$ we have that

$$
t \mapsto \int_{\Omega(t)} u(x, t) v(x, t) d x
$$

is continuous in $[0, T]$ and there is a constant $c$, depending only on $T$, such that

$$
\max _{t \in[0, T]} \int_{\Omega(t)} u(x, t)^{2} d x \leq c\|u\|_{\mathcal{W}}
$$

Proof. First we show that the function $t \mapsto \int_{\Omega(t)} u(x, t) v(x, t) d x$ is bounded. Notice that, by the absolute continuity of $t \mapsto \int_{\Omega(t)} d x$, the function above is surely measurable for $u, v \in C^{0}(\bar{Q})$. Notice that formula (5) holds also if we consider $u, v \in \tilde{\mathcal{U}}$, the space defined reflecting the functions in time as follows: first define

$$
\tilde{Q}:=\hat{Q} \cup \bigcup_{t \in[-T, 0]}(\Omega(-t) \times\{t\})
$$


and for every $u \in \mathcal{U}$ consider a function $\tilde{u}$ defined in $\tilde{Q}$ as follows

$$
\tilde{u}(x, t)= \begin{cases}u(x, t) & \text { for } t \geq 0 \\ u(x,-t) & \text { for } t \leq 0\end{cases}
$$

and denote by $\tilde{\mathcal{U}}$ the space of such $\tilde{u}$. Now if we fix $t \in[0, T]$, consider $\varphi \in$ $C^{1}([-T, T] ; \mathbf{R})$ such that $\varphi(-T)=0, \varphi(s)=1$ for $s \in[0, t], 0 \leq \varphi \leq 1,0 \leq$ $\varphi^{\prime} \leq 2 / T$, consider $\tilde{u}, \tilde{v} \in \tilde{\mathcal{U}}$ and notice that $\varphi \tilde{u}$ belongs to $\tilde{\mathcal{U}}$. Apply (5) to $(\varphi \tilde{u}) \tilde{v}$ with $t_{1}=-T, t_{2}=t$ to get that $\partial_{t}(\varphi \tilde{u} \tilde{v})=\tilde{u} \partial_{t} \tilde{v} \varphi+\tilde{v} \partial_{t} \tilde{u} \varphi+\tilde{u} \tilde{v} \varphi^{\prime}$ and get

$$
\begin{aligned}
\left|\int_{\Omega(t)} u(x, t) v(x, t) d x\right| & =\left|\int_{-T}^{t} \int_{\Omega(s)}\left[\tilde{u} \partial_{t} \tilde{v} \varphi+\tilde{v} \partial_{t} \tilde{u} \varphi+\tilde{u} \tilde{v} \varphi^{\prime}\right] d x d s\right| \\
& \leq \int_{-T}^{T} \int_{\Omega(s)}\left[\left|\tilde{u} \partial_{t} \tilde{v} \varphi\right|+\left|\tilde{v} \partial_{t} \tilde{u} \varphi\right|+\left|\tilde{u} \tilde{v} \varphi^{\prime}\right|\right] d x d s \\
& \leq 2 \int_{0}^{T} \int_{\Omega(s)}\left[|u|\left|\partial_{t} v\right|+\left|v \| \partial_{t} v\right|+u v \varphi^{\prime}\right] d x d s \\
& \leq\|u\|_{\mathcal{V}}^{2}+\left\|v_{t}\right\|_{\mathcal{V}^{\prime}}^{2}+\|v\|_{\mathcal{V}}^{2}+\left\|u_{t}\right\|_{\mathcal{V}^{\prime}}^{2}+\frac{2}{T}\left(\|u\|_{\mathcal{H}}^{2}+\|v\|_{\mathcal{H}}^{2}\right) \\
& \leq \tilde{c}\left(\|u\|_{\mathcal{V}}^{2}+\|v\|_{\mathcal{V}}^{2}\right)
\end{aligned}
$$

where $\tilde{c}=\tilde{c}(T)$. In particular taking $u=v$ we have $(c=2 \tilde{c})$

$$
\sup _{t \in(0, T)} \int_{\Omega(t)} u(x, t)^{2} d x \leq c\|u\|_{\mathcal{W}}^{2}
$$

Moreover, taking $u=v$ in (5), $u \in \mathcal{U}$ and letting $t_{1} \rightarrow t_{2}$ we also get that

$$
t \mapsto \int_{\Omega(t)} u(x, t)^{2} d x \text { is continuous in }[0, T]
$$

By inequality (6) and by the density of $\mathcal{U}$ in $\mathcal{W}$ we also get that the embedding (as done in Problem 23.10 in [10])

$$
j: \mathcal{U} \rightarrow \mathcal{X}:=\left\{u \in \mathcal{V} \mid t \mapsto \int_{\Omega(t)} u(x, t)^{2} d x \text { is continuous in }[0, T]\right\}
$$

has a unique extension $j: \mathcal{W} \rightarrow \mathcal{X}$ (see Proposition 18.29 in [10]) and in this sense (7) holds.

To conclude we observe that indeed we have that a function $u \in \mathcal{W}$ belongs to $C^{0}([0, T] ; H)$, where $H=L^{2}(A)$ and $A \supset \Omega(t)$ for very $t \in[0, T]$, and $u(\cdot, t) \in H_{0}^{1}(\Omega(t))$ for almost every $t \in[0, T]$. Then 


$$
\begin{aligned}
& \left|\int_{\Omega\left(t_{2}\right)} u\left(x, t_{2}\right) v\left(x, t_{2}\right) d x-\int_{\Omega\left(t_{1}\right)} u\left(x, t_{1}\right) v\left(x, t_{1}\right) d x\right| \\
& =\left|\int_{A} u\left(x, t_{2}\right) v\left(x, t_{2}\right) d x-\int_{A} u\left(x, t_{1}\right) v\left(x, t_{1}\right) d x\right| \\
& =\mid \int_{A} u\left(x, t_{2}\right) v\left(x, t_{2}\right) d x-\int_{A} u\left(x, t_{2}\right) v\left(x, t_{1}\right) d x \\
& \quad+\int_{A} u\left(x, t_{2}\right) v\left(x, t_{1}\right) d x-\int_{A} u\left(x, t_{1}\right) v\left(x, t_{1}\right) d x \mid \\
& \leq\|u\|_{C^{0}([0, T] ; H)}\left\|v\left(t_{2}\right)-v\left(t_{1}\right)\right\|_{H}+\|v\|_{C^{0}([0, T] ; H)}\left\|u\left(t_{2}\right)-u\left(t_{1}\right)\right\|_{H}
\end{aligned}
$$

by which we also get that $t \mapsto \int_{\Omega(t)} u(x, t) v(x, t) d x$ is continuous.

Proposition 2.6. For every $u, v \in \mathcal{W}$ we have that

$$
\left.\frac{d}{d t}\right|_{t=s} \int_{\Omega(t)} u(x, t) v(x, t) d x=\left\langle u_{t}(s), v(s)\right\rangle_{V^{\prime}(s) \times V(s)}+\left\langle v_{t}(s), u(s)\right\rangle_{V^{\prime}(s) \times V(s)},
$$

and for $t_{1}, t_{2} \in[0, T]$

$$
\begin{aligned}
& \int_{t_{1}}^{t_{2}}\left[\left\langle u_{t}(s), v(s)\right\rangle_{V^{\prime}(s) \times V(s)}+\left\langle v_{t}(s), u(s)\right\rangle_{V^{\prime}(s) \times V(s)}\right] d s \\
& \quad=\int_{\Omega\left(t_{2}\right)} u\left(x, t_{2}\right) v\left(x, t_{2}\right) d x-\int_{\Omega\left(t_{1}\right)} u\left(x, t_{1}\right) v\left(x, t_{1}\right) d x .
\end{aligned}
$$

Proof. The formulas stated in the proposition are clearly true for $u, v \in \mathcal{U}$. We want to show they are true also for $u, v \in \mathcal{W}$. The proof follows by density of $\mathcal{U}$ in $\mathcal{W}$ and by formula (5). We have the two triplets

$$
\begin{aligned}
& \mathcal{V} \subset \mathcal{H} \subset \mathcal{V}^{\prime} \\
& \mathcal{V}_{A}:=L^{p}(0, T ; V) \subset L^{2}(0, T ; H) \subset L^{p^{\prime}}\left(0, T ; V^{\prime}\right)=: \mathcal{V}_{A}^{\prime}
\end{aligned}
$$

where we have defined $V:=W_{0}^{1, p}(A), H=L^{2}(A), V^{\prime}=W^{-1, p^{\prime}}(A)$, and notice that $\mathcal{V}$ continuously embeds in $\mathcal{V}_{A}$ and is a closed subspace of $\mathcal{V}_{A}$ (clearly by extending functions in $\mathcal{V}$ by 0 to $(A \times[0, T]) \backslash Q)$. Since $\mathcal{V}$ is a closed and proper subspace of $\mathcal{V}_{A}$ also every linear and continuous form $f \in \mathcal{V}^{\prime}$ can be extended to an element of $\mathcal{V}_{A}^{\prime}$, again extending by 0 outside of $Q$. Indeed, denoting by $\bar{u}$ the extension to $\mathcal{V}_{A}$ of a function $u \in \mathcal{V}$, i.e.

$$
\bar{u}=u \quad \text { in } Q, \quad \bar{u}=0 \quad \text { in } A \times(0, T) \backslash Q,
$$

we get $(\bar{u})_{t} \in \mathcal{V}_{A}^{\prime}$. This can be easily seen since $u_{t}$ can be represented, in a unique way, by $n+1$ functions $f_{0}, f_{1}, \ldots f_{n} \in L^{p^{\prime}}(Q)$ such that (see, e.g., Theorem 3.8 in [3])

$$
\begin{aligned}
& \left\langle u_{t}, v\right\rangle=\int_{0}^{T} \int_{A} f_{0} v d x d t+\sum_{j=1}^{n} \int_{0}^{T} \int_{A} f_{j} D_{j} v d x d t \\
& \text { and }\left\|u_{t}\right\|_{\mathcal{V}^{\prime}}=\left\|\left(f_{0}, f_{1}, \ldots f_{n}\right)\right\|_{\left(L^{p^{\prime}}(Q)\right)^{n+1}} .
\end{aligned}
$$

Then $(\bar{u})_{t}$ is analogously (and uniquely) represented by $\left(\bar{f}_{0}, \bar{f}_{1}, \ldots \bar{f}_{n}\right)$. 
We conclude that if $u \in \mathcal{W}$ we have that $\bar{u} \in \mathcal{W}_{A}=\left\{u \in \mathcal{V}_{A} \mid u_{t}=u^{\prime} \in\right.$ $\left.\mathcal{V}_{A}^{\prime}\right\}$ and we define

$$
\int_{0}^{T}\left\langle u_{t}(s), v(s)\right\rangle_{V^{\prime}(s) \times V(s)} d s:=\int_{0}^{T}\left\langle(\bar{u})_{t}(s), \bar{v}(s)\right\rangle_{V^{\prime} \times V} d s .
$$

Now, by a standard procedure, using the density of $\mathcal{U}$ in $\mathcal{W}$ and using the right hand side of the last equality, we can conclude (see also Problem 23.10 in $[10])$.

Before ending this section we recall some classical definitions and a classical result we will apply in the next section. By $X$ we denote a Banach space endowed with the norm $\|\cdot\|$, and by $\langle\cdot, \cdot\rangle$ the duality between its dual space $X^{\prime}$ and $X$.

Definition 2.7. We say that an operator $B: X \rightarrow X^{\prime}$ is coercive if

$$
\lim _{\|x\| \rightarrow+\infty} \frac{\langle B x, x\rangle}{\|x\|} \rightarrow+\infty,
$$

is bounded if it maps a bounded set in a bounded set, is pseudomonotone if

$$
x_{n} \rightarrow x \text { in } X \text {-weak and } \quad \limsup _{n}\left\langle B x_{n}, x_{n}-x\right\rangle \leq 0
$$

implies that

$$
\langle B x, x-y\rangle \leq \liminf _{n}\left\langle B x_{n}, x_{n}-y\right\rangle \quad \text { for every } y \in X .
$$

Finally we say that $B$ is monotone if $\langle B x-B y, x-y\rangle \geq 0$ for every $x, y \in X$ and strictly monotone if $\langle B x-B y, x-y\rangle>0$ for every $x, y \in X$ with $x \neq y$.

We recall now a classical result (see, for instance, Section 32.4 in [10]).

Theorem 2.8. Let $A: X \rightarrow X^{\prime}$ ( $X^{\prime}$ the dual space of $X, X$ Banach space) be pseudomonotone, bounded and coercive. Suppose $L: X \rightarrow 2^{X^{\prime}}$ to be maximal monotone. Then for every $f \in X^{\prime}$ the following equation has a solution

$$
L u+A u \ni f
$$

and in particular if $L, A$ are single-valued the equation $L u+A u=f$ has a solution.

\section{The existence result}

In this section we want to prove a general existence result for an evolution equation. Thanks to Proposition 2.5 we can define

$$
\mathcal{W}^{0}=\{u \in \mathcal{W} \mid u(x, 0)=0 \text { in } \Omega(0)\}
$$

and then consider the operator

$$
\mathcal{L} u=u_{t}, \quad D(\mathcal{L})=\mathcal{W}^{0} .
$$

Proposition 3.1. The operator $\mathcal{L}: D(\mathcal{L}) \subset \mathcal{V} \rightarrow \mathcal{V}^{\prime}$ is maximal monotone. 
Proof. For simplicity we will denote by $\langle\cdot, \cdot\rangle$ the quantity $\langle\cdot, \cdot\rangle_{\mathcal{V}^{\prime} \times \mathcal{V}}$. The first step

$$
\langle\mathcal{L} u, u\rangle \geq 0
$$

follows from Proposition 2.6 since for $u \in \mathcal{W}^{0}$

$$
\langle\mathcal{L} u, u\rangle=\frac{1}{2} \int_{\Omega(T)} u^{2}(x, T) d x-\frac{1}{2} \int_{\Omega(0)} u^{2}(x, 0) d x \geq 0 .
$$

To see that it is maximal monotone fix $w \in \mathcal{V}^{\prime}$ and $v \in \mathcal{V}$ and suppose

$$
\langle w-\mathcal{L} u, v-u\rangle \geq 0
$$

for every $u \in D(\mathcal{L})$. We want to show that $v \in D(\mathcal{L})$ and $w=\mathcal{L} v$. If we define $z=v-u$ we obtain that

$$
\langle w, z\rangle-\langle\mathcal{L}(v-z), z\rangle \geq 0 .
$$

Fix $z$ and consider $\lambda z$ with $\lambda \in \mathbf{R}$. Since $\mathcal{L}$ is linear for every positive $\lambda$ we obtain

$$
\lambda\langle\mathcal{L} z, z\rangle \geq\langle\mathcal{L} v-w, z\rangle
$$

and for every negative $\lambda$

$$
\lambda\langle\mathcal{L} z, z\rangle \leq\langle\mathcal{L} v-w, z\rangle
$$

for every $z \in v+D(\mathcal{L})$. Letting $\lambda$ go to zero we obtain that $\langle w-\mathcal{L} v, z\rangle=0$, i. e.

$$
\langle w-\mathcal{L} v, v\rangle=\langle w-\mathcal{L} v, u\rangle
$$

for every $u \in D(\mathcal{L})$ which implies $\langle w-\mathcal{L} v, u\rangle=0$ for every $u \in D(\mathcal{L})$. By the density of $\mathcal{W}^{0}$ in $\mathcal{V}$ we get that

$$
\mathcal{L} v=w \text { in } \mathcal{V}^{\prime}
$$

Then, since $v \in \mathcal{V}$ and $w \in \mathcal{V}^{\prime}$ and $\mathcal{L} v=v_{t}$ we conclude that $v \in \mathcal{W}$. We have to see now that $v \in D(\mathcal{L})$. For every $u \in D(\mathcal{L})$ we have that

$$
\left.2\langle\mathcal{L}(v-u), v-u\rangle=\int_{\Omega(T)}(v(x, T)-u(x, T))^{2} d x-\int_{\Omega(0)} v(x, 0)\right)^{2} d x
$$

It is sufficient to consider $u \in D(\mathcal{L})$ satisfying $u(x, T)=v(x, T)$ to get

$$
\left.-\int_{\Omega(0)} v(x, 0)\right)^{2} d x \geq 0
$$

which implies that $v(x, 0)=0$ in $\Omega(0)$ which concludes the proof.

Now consider an operator $\mathcal{A}: \mathcal{V} \longrightarrow \mathcal{V}^{\prime}$ satisfying the following assumptions:

$\mathcal{A}$ pseudomonotone, coercive, bounded (see Definition 2.7). 
Definition 3.2. Given $f \in \mathcal{V}^{\prime}, u_{o} \in L^{2}(\Omega(0))$ we say solution of the problem

$$
\begin{cases}u_{t}+\mathcal{A} u=f & \text { in } Q \\ u=0 & \text { in } \Gamma \\ u(0)=u_{o} & \text { in } \Omega(0),\end{cases}
$$

a function $u \in \mathcal{W}$ such that $u_{t}+\mathcal{A} u=f$ in $\mathcal{V}^{\prime}$ and $u(x, 0)=u_{o}(x)$ in $L^{2}(\Omega(0))$. If $\Omega(0)=\emptyset$ the initial condition has no meaning.

Remark 3.3. If $\Omega(0)=\emptyset$ the problem can be simply written

$$
\begin{cases}u_{t}+\mathcal{A} u=f & \text { in } Q \\ u=0 & \text { in } \Gamma,\end{cases}
$$

even if we continue to write $u(0)=u_{o}$ in $\Omega(0)$ just to consider the general case.

Now we start considering the following problem

$$
\begin{cases}u_{t}+\mathcal{A} u=f & \text { in } Q \\ u=0 & \text { in } \Gamma \\ u(0)=0 & \text { in } \Omega(0),\end{cases}
$$

Theorem 3.4. Suppose $\mathcal{A}$ pseudomonotone, coercive, bounded, according to Definition 2.7. Then problems (11) admit a solution for every $f \in \mathcal{V}^{\prime}$. If moreover $\mathcal{A}$ is strictly monotone the solution is unique.

Remark 3.5. In fact we obtain an existence result also for the Cauchy problem

$$
u_{t}+\mathcal{A} u \ni f, \quad u \in \mathcal{W}^{0} .
$$

Moreover the solution is unique if the operator $\mathcal{A}$ of Theorem 3.4 is strictly monotone.

Proof. By Theorem 2.8 (see for more details Theorem 32.A, Corollary 32.25, Corollary 32.26 and also Proposition 27.7 in [10]) and Proposition 3.1 we obtain the existence.

As regards uniqueness it is sufficient to observe that if $u, v$ are two solutions we have

$$
0=\left\langle(u-v)_{t}+\mathcal{A} u-\mathcal{A} v, u-v\right\rangle \geq\langle\mathcal{A} u-\mathcal{A} v, u-v\rangle \geq 0 .
$$

Since $\mathcal{A}$ is strictly monotone we conclude that $u=v$.

Now we give some a priori estimates supposing the operator $\mathcal{A}$ satisfies some standard growth conditions.

Theorem 3.6. Suppose there exist two positive constants $\alpha, \beta>0$ such that for some $p \in[2,+\infty)$

$$
\langle\mathcal{A} u-\mathcal{A} v, u-v\rangle_{\mathcal{V}^{\prime} \times \mathcal{V}} \geq \alpha\|u-v\|_{\mathcal{V}}^{p}, \quad\|\mathcal{A} u\|_{\mathcal{V}^{\prime}} \leq \beta\|u\|_{\mathcal{V}}^{p-1}
$$

for every $u, v \in \mathcal{V}$. Then, defined the operator $\mathcal{P}: \mathcal{W} \rightarrow \mathcal{V}^{\prime}$ by $\mathcal{P} u=u^{\prime}+\mathcal{A} u$, the following estimates hold: there is a constant $c=c(\alpha, \beta, p)$ (depending only on $\alpha, \beta$ and $p$ ) such that for every $u \in \mathcal{W}$

$$
\|u\|_{\mathcal{W}} \leq c\left[\|\mathcal{P} u\|_{\mathcal{V}^{\prime}}+\|\mathcal{P} u\|_{\mathcal{V}^{\prime}}^{1 /(p-1)}+\|u(0)\|_{H(0)}^{2 / p}\right]
$$


Moreover for every $f \in \mathcal{V}^{\prime}$ and $u_{o} \in H(0)=L^{2}(\Omega(0)$ ) problem (9) has a unique solution.

Proof. We estimate $(q=p /(p-1))$, for every $\epsilon>0$,

$$
\|\mathcal{P} u\|_{\mathcal{V}^{\prime}}\|u\|_{\mathcal{V}} \leq \frac{\epsilon^{p}}{p}\|u\|_{\mathcal{V}}^{p}+\frac{1}{q}\left(\frac{1}{\epsilon}\right)^{q / p^{2}}\|\mathcal{P} u\|_{\mathcal{V}^{\prime}}^{q}
$$

and, by Proposition 2.6,

$$
\begin{aligned}
2\left\langle u_{t}, u\right\rangle & =(u(T), u(T))_{H(T)}-(u(0), u(0))_{H(0)} \\
& =\int_{\Omega(T)} u^{2}(x, T) d x-\int_{\Omega(0)} u^{2}(x, 0) d x .
\end{aligned}
$$

Then, since $\langle\mathcal{A} u, u\rangle=\langle\mathcal{P} u, u\rangle-\left\langle u_{t}, u\right\rangle$, we have

$$
\alpha\|u\|_{\mathcal{V}}^{p} \leq\|\mathcal{P} u\|_{\mathcal{V}^{\prime}}\|u\|_{\mathcal{V}}+\frac{1}{2}\left[(u(0), u(0))_{H(0)}-(u(T), u(T))_{H(T)}\right]
$$

and using (13) for a suitable and little enough value of $\epsilon$ (which can be chosen depending only on $\alpha$ and $p$ ) we infer

$$
\|u\|_{\mathcal{V}} \leq c(\alpha, p)\left[\|\mathcal{P} u\|_{\mathcal{V}^{\prime}}^{p /(p-1)}+(u(0), u(0))_{H(0)}-(u(T), u(T))_{H(T)}\right]^{1 / p} .
$$

We conclude estimating $\left\|u_{t}\right\|$ as follows: since $u_{t}=\mathcal{P} u-\mathcal{A} u$ we obtain that $\left\|u_{t}\right\|_{\mathcal{V}^{\prime}} \leq\|\mathcal{P} u\|_{\mathcal{V}^{\prime}}+\beta\|u\|_{\mathcal{V}}^{p-1}$

$$
\|u\|_{\mathcal{W}} \leq c(\alpha, \beta, p)\left[\|\mathcal{P} u\|_{\mathcal{V}^{\prime}}+\|\mathcal{P} u\|_{\mathcal{V}^{\prime}}^{1 /(p-1)}+\|u(0)\|_{H(0)}^{2 / p}\right] .
$$

As regards the existence and uniqueness of the solution notice that thanks to Proposition 2.5 every function $u \in \mathcal{W}$ is such that $u(\cdot, t) \in L^{2}(\Omega(t))$ for every $t \in[0, T]$. In particular we can choose a function $w \in \mathcal{W}$ in such a way that $w(\cdot, 0)=u_{o}$ in $L^{2}(\Omega(0))$. By Theorem 3.4 the problem

$$
\begin{cases}v_{t}+\mathcal{A}(v+w)=f-w_{t} & \text { in } Q \\ v=0 & \text { in } \Gamma \\ v(0)=0 & \text { in } \Omega(0),\end{cases}
$$

has a unique solution $v$. Indeed the operator $\tilde{\mathcal{A}}(v):=\mathcal{A}(v+w)$ is bounded and pseudomonotone. Moreover if we suppose (12) we see that $\tilde{\mathcal{A}}$ is also coercive:

$$
\langle\tilde{\mathcal{A}} v, v\rangle=\langle\mathcal{A}(v+w)-\mathcal{A} w, v\rangle+\langle\mathcal{A} w, v\rangle .
$$

Dividing by $\|v\|$ we obtain for the second term that

$$
\left|\frac{\langle\mathcal{A} w, v\rangle}{\|v\|}\right| \leq\|\mathcal{A} w\|
$$

and for the first, by the monotonicity of $\mathcal{A}$,

$$
\frac{\langle\mathcal{A}(v+w)-\mathcal{A} w, v+w-w\rangle}{\|v\|} \geq \alpha\|v\|^{p-1} .
$$

Then the function $u=v+w$ satisfies (9). 
Remark 3.7. If $\mathcal{A}$ is linear we also have the corresponding existence result for the problem

$$
\begin{cases}u_{t}+\mathcal{A} u+\lambda u=f & \text { in } Q \\ u=0 & \text { in } \Gamma \\ u(0)=u_{o} & \text { in } \Omega(0),\end{cases}
$$

for every $\lambda \in \mathbf{R}$. It is sufficient indeed to consider the change of variable $v(x, t)=e^{\lambda t} u(x, t)$ to obtain

$$
\begin{cases}v_{t}+\mathcal{A} v=\tilde{f}=f e^{\lambda t} & \text { in } Q \\ v=0 & \text { in } \Gamma \\ v(0)=u_{o} & \text { in } \Omega(0),\end{cases}
$$

which has a unique solution $v$. Then $u(x, t)=v(x, t) e^{-\lambda t}$ solves the original problem.

\section{Examples}

Here we give some simple example showing some pictures representing some possible sets $Q$ in which Eq. (1) holds. We confine to draw some pictures in $\mathbf{R}^{2}$. In the following pictures the vertical axis represents the variable $t$. In the following we will denote by $\mathcal{A}$ an operator satisfying the assumptions made in Theorem 3.6. In this section we want to focus our attention on the possible sets $Q$ in which problem (9) is solvable with attention to assumption (H.2). The set $Q$ will be contained in $A \times[S, T]$ for some open set $A$.

1. In the first example the set $Q$ (see Fig. 1) is a subset of $\mathbf{R}^{2}$ of the following type:

$$
Q=\left\{(x, t) \in \mathbf{R}^{2} \mid t \in[S, T], \gamma_{1}(t) \leq x \leq \gamma_{2}(t)\right\} .
$$

In this simple case $\Omega(t)$ turns out to be the interval $\left(\gamma_{1}(t), \gamma_{2}(t)\right)$. The existence of a family of maps $G^{t_{o}}$ required in assumption (H.2) becomes the existence of only one map $G:[a, b] \times\left[T_{0}, T_{1}\right] \rightarrow Q$ where, for instance, one can choose $a=\gamma_{1}\left(T_{0}\right), b=\gamma_{2}\left(T_{0}\right)$. A choice for the map $G$ can be the following:

$$
G(x, t)=\frac{x-a}{b-a}\left(\gamma_{2}(t)-\gamma_{1}(t)\right)+\gamma_{1}(t)
$$

Then it is quite easy to see that the functions

$$
\begin{aligned}
t & \mapsto \int_{\Omega(t)} d x=\int_{\gamma_{1}(t)}^{\gamma_{2}(t)} d x, \\
t & \mapsto G(x, t), \quad t \mapsto D G(x, t) \quad \text { for (almost) every } x \in[a, b]
\end{aligned}
$$

are absolutely continuous, and then our assumptions (H.1) and (H.2) are satisfied, if the profiles $\gamma_{1}$ and $\gamma_{2}$ are absolutely continuous.

A simple example in higher dimension could be a set $Q$ whose profile is obtained rotating the graph of an absolute continuous function around the vertical axis.

2. In the second example the set $Q$ is a connected subset of $A \times[S, T]$, being $A$ an open set containing $\Omega(t)$ for every $t$, but we suppose there is $t_{o} \in(S, T)$ 


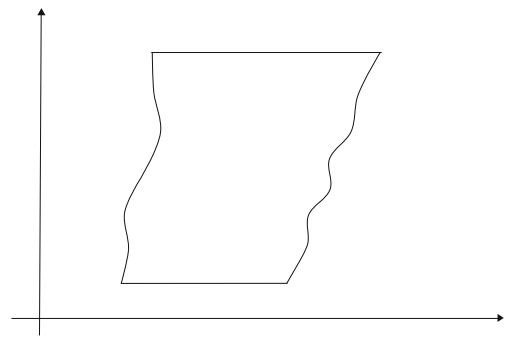

FIGURE 1.

such that $\Omega(t)$ is not connected, but may be the union of two (or more) disjoint and connected sets. For simplicity, as shown in Fig. 2 (where $\Omega(t)$ is an interval or the union of two disjoin intervals) we suppose to have $S_{1}, S_{2}, t_{o}$ with

$$
S_{1}=S, \quad S_{1}<S_{2}<t_{o}
$$

We denote by $\Omega_{1}(t)$ and $\Omega_{2}(t)$ the two potential connected components of $\Omega(t)$ for $t \in\left[S, t_{o}\right)$. For $t \in\left[S_{1}, S_{2}\right)$ the set $\Omega_{2}(t)$ is the empty set and $\Omega(t)=\Omega_{1}(t)$, while for $t \in\left[S_{2}, t_{o}\right)$ we have

$$
\Omega(t)=\Omega_{1}(t) \cup \Omega_{2}(t), \quad \Omega_{1}(t) \neq \emptyset, \quad \Omega_{2}(t) \neq \emptyset .
$$

Once defined $Q^{-}$and $Q^{+}$the sets

$$
Q^{-}:=Q \cap\left(A \times\left(S_{1}, t_{o}\right)\right), \quad Q^{+}:=Q \cap\left(A \times\left(t_{o}, T\right)\right),
$$

also the set $Q^{-}$turns out to be not connected and is the union of $Q_{1}^{-}$and $Q_{2}^{-}$ where

$$
Q_{1}^{-}=\cup_{t \in\left(S_{1}, t_{o}\right)} \Omega_{1}(t), \quad Q_{2}^{-}=\cup_{t \in\left(S_{2}, t_{o}\right)} \Omega_{2}(t) .
$$

We define moreover

$$
\Gamma_{1}^{-}:=\cup_{t \in\left(S_{1}, t_{o}\right)} \partial \Omega_{1}(t), \quad \Gamma_{2}^{-}:=\cup_{t \in\left(S_{2}, t_{o}\right)} \partial \Omega_{2}(t) .
$$

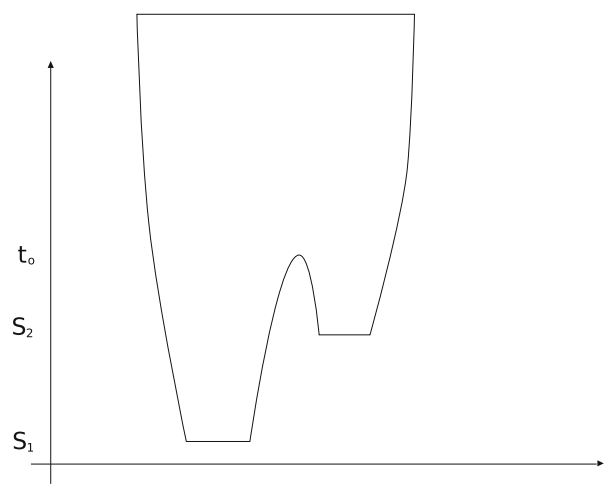

FiguRE 2. 
Assumptions (H.1) and (H.2) can be satisfied even if the sections $\Omega(t)$ are disconnected for $t<t_{o}$ and connected for $t>t_{o}$. Then the problem

$$
(\mathrm{P}) \quad \begin{cases}u_{t}+\mathcal{A} u=f & \text { in } Q \\ u=0 & \text { in } \Gamma \\ u\left(x, S_{1}\right)=u_{1}(x) & \text { in } \Omega_{1}\left(S_{1}\right) \\ u\left(x, S_{2}\right)=u_{2}(x) & \text { in } \Omega_{2}\left(S_{2}\right),\end{cases}
$$

has a (unique) solution. In general one could consider as many "initial conditions" as the connected components of $\Omega(t)$.

To show that the problem above has a unique solution one can solve the two following problems
I) $\begin{cases}u_{t}+\mathcal{A} u=f & \text { in } Q_{1}^{-} \\ u=0 & \text { in } \Gamma_{1}^{-} \\ u\left(x, S_{1}\right)=u_{1}(x) & \text { in } \Omega_{1}\left(S_{1}\right),\end{cases}$
II) $\begin{cases}u_{t}+\mathcal{A} u=f & \text { in } Q_{2}^{-} \\ u=0 & \text { in } \Gamma_{2}^{-} \\ u\left(x, S_{2}\right)=u_{2}(x) & \text { in } \Omega_{2}\left(S_{1}\right),\end{cases}$

denote by $u_{1}^{-}$the solution of problem I) and by $u_{2}^{-}$the solution of problem II), solve the third problem

$$
\text { III) } \begin{cases}u_{t}+\mathcal{A} u=f & \text { in } Q^{+} \\ u=0 & \text { in } \Gamma^{+} \\ u\left(x, t_{o}\right)=v(x) & \text { in } \Omega\left(t_{o}\right),\end{cases}
$$

where

$$
\Omega\left(t_{o}\right)=\Omega_{1}\left(t_{o}\right) \cup \Omega_{2}\left(t_{o}\right), \quad v(x):= \begin{cases}u_{1}\left(x, t_{o}\right) & \text { for } x \in \Omega_{1}\left(t_{o}\right) \\ u_{2}\left(x, t_{o}\right) & \text { for } x \in \Omega_{2}\left(t_{o}\right),\end{cases}
$$

and denote by $u^{+}$the solution of problem III). The solution $u$ of problem (P) is then defined as

$$
u=u_{1}^{-} \text {in } Q_{1}^{-}, \quad u=u_{2}^{-} \text {in } Q_{2}^{-}, \quad u=u^{+} \text {in } Q^{+} .
$$

3. We can also consider problems without a real initial conditions. For example a set $Q$ like that in Fig. 3 may be considered and, provided that assumptions (H.1) and (H.2) are satisfied, one can consider the problem

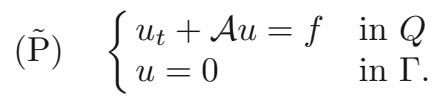

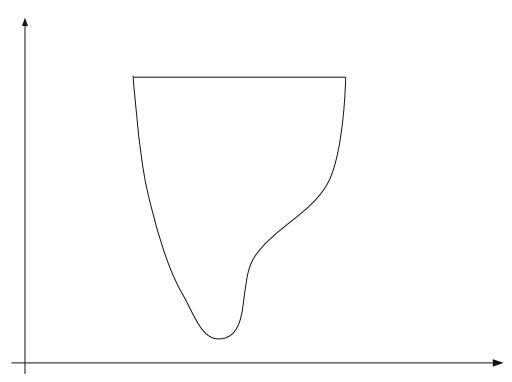

FiguRE 3. 

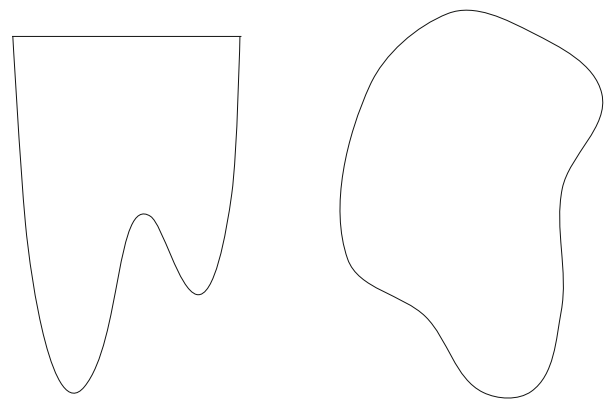

\section{Figure 4.}

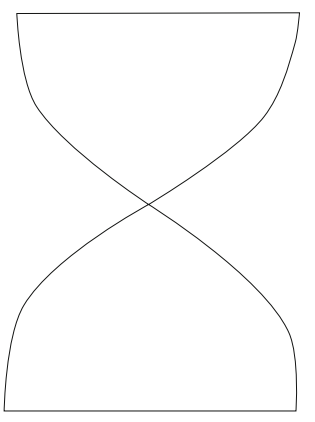

FiguRE 5 .

If $Q=\cup_{t \in(S, T)} \Omega(t)$ we suppose that $\Omega(S)=\emptyset$ and $\Omega(t) \neq \emptyset$ for $t>S$. In fact the initial condition is contained in the boundary condition in $\Gamma$.

4. Arguing as in the example 2. and as in the example 3. one could also consider problem $(\tilde{\mathrm{P}})$ with $Q$ like in Fig. 4 in which no initial condition is given. Also a set like that in Fig. 5 could be considered, i.e. a set where $\Omega(t)$ can become empty for some $t_{o} \in(S, T)$.

Even in this case one can treat the problem as two different problems: one for $t \in\left[S, t_{o}\right]$ and another for $t \in\left[t_{o}, T\right]$.

\section{Acknowledgments}

The author wish to thank the anonymous referees for many useful suggestions by which the paper turns out to be markedly improved.

\section{References}

[1] Abdulla, U.G.: Well-posedness of the Dirichlet problem for the non-linear diffusion equation in non-smooth domains. Trans. Am. Math. Soc. 357, 247-265 (2005, electronic) 
[2] Acquistapace, P., Terreni, B.: A unified approach to abstract linear nonautonomous parabolic equations. Rend. Sem. Mat. Univ. Padova 78, 47-107 (1987)

[3] Adams, R.A.: Sobolev Spaces. Academic Press, New York (1975)

[4] Attouch, H., Damlamian, A.: Problèmes d'évolution dans les Hilberts et applications. J. Math. Pures Appl. 54(9), 53-74 (1975)

[5] Bonaccorsi, S., Guatteri, G.: A variational approach to evolution problems with variable domains. J. Differ. Equ. 175, 51-70 (2001)

[6] Delfour, M.C., Zolésio, J.-P.: Shapes and Geometries. Advances in Design and Control. Metrics, Analysis, Differential Calculus, and Optimization, vol. 22, 2nd edn. Society for Industrial and Applied Mathematics (SIAM), Philadelphia (2011)

[7] Gianazza, U., Savaré, G.: Abstract evolution equations on variable domains: an approach by minimizing movements. Ann. Scuola Norm. Sup. Pisa Cl. Sci. 23(4), 149-178 (1996)

[8] Gilbarg, D., Trudinger, N.S.: Elliptic Partial Differential Equations of Second Order. Springer, Berlin (1977)

[9] Lions, J.L.: Équations Linéaires du $1^{\mathrm{er}}$ Ordre. Equazioni Differenziali Astratte. C.I.M.E. Seminar, vol. 29, pp. 15-28 (1963)

[10] Zeidler, E.: Nonlinear Functional Analysis and Its Applications, vol. II A and II B. Springer, New York (1990)

Fabio Paronetto

Dipartimento di Matematica

Università di Padova

via Trieste 63

35121 Padua

Italy

e-mail: fabio.paronetto@unipd.it

Received: 10 April 2012.

Accepted: 26 February 2013. 